\title{
Telangiectasia hemorrágica hereditaria: A propósito de un caso clínico
}

\author{
Hereditary hemorrhagic telangiectasia: On the subject of a clinical case
}

\author{
Mario Alvarez $\mathrm{T}^{1}$, Mackarena Otárola $\mathrm{M}^{2}$, Tamara Novoa 0², Daniel Moyano $\mathbf{M}^{1}$, Luis Cabezas $\mathrm{C}^{1}$.
}

\begin{abstract}
RESUMEN
La epistaxis, es un síntoma frecuente en la consulta de otorrinolaringología. Dentro de las causas posibles encontramos la telangiectasia hemorrágica hereditaria (síndrome de Rendu Osler Weber), que corresponde a un desorden autosómico dominante caracterizado por sangrados nasales y gastrointestinales asociados a malformaciones arteriovenosas sistémicas. Su manifestación más frecuente es la epistaxis, presentándose más frecuentemente en personas mayores de 40 años, sin predilección por género. Se presenta el caso de un paciente de sexo masculino de 46 años quien consulta por epistaxis a repetición y severa. Durante la hospitalización se efectúa el tratamiento convencional de la epistaxis, diagnóstico retroactivo del síndrome de Rendu Osler Weber y manejo multidisciplinario de la patología. Se realiza revisión de la literatura y discusión del manejo del paciente que cursa con esta enfermedad.
\end{abstract}

Palabras clave: Telangiectasia hemorrágica familiar, síndrome de Rendu Osler Weber, malformación arterio-venosa.

\section{ABSTRACT}

Epistaxis is a common symptom in the otorhinolaryngology consultation. Among the possible causes are hereditary hemorrhagic telangiectasia (Rendu Osler Weber syndrome), which corresponds to an autosomal dominant disorder characterized by nasal and gastrointestinal bleeding associated with systemic arteriovenous malformations. Its most frequent manifestation is epistaxis, presenting more frequently in people over 40 years of age, without gender preference. We present the case of a male patient of 46 years old who consults for recurrence and severe epistaxis. During hospitalization, conventional treatment of epistaxis, retroactive diagnosis of Rendu Osler Weber syndrome and multidisciplinary management of pathology are performed. We review the literature and discuss the management of patients with this disease.

Key words: Hereditary hemorrhagic telangiectasia, Rendu Osler Weber Syndrome Arterio-Venous Malformation.

\footnotetext{
1 Médicos Servicio de Otorrinolaringología del Hospital Carlos van Buren.

2 Interna de Medicina Universidad de Valparaíso.
} 


\section{INTRODUCCIÓN}

La epistaxis o sangrado de origen nasal es un motivo de consulta frecuente en otorrinolaringología, estimándose que el $60 \%$ de la población sufre algún grado de epistaxis en su vida; sólo el $6 \%$ de ellos requerirá atención médica para tratamiento y control de su hemostasia, y 1,6 por cada 100.000 pacientes serán hospitalizados ${ }^{1}$. Incluye una gran variedad de causas, tanto locales como sistémicas y estas mismas van variando en frecuencia e importancia en relación a la edad del paciente. Dentro de éstas podemos identificar un grupo de patologías que pueden ser manifiestas desde cualquier edad, siendo más frecuente en adultos, correspondientes a un síndrome vasculopático, debido a malformaciones congénitas, destacando dentro de éstas a la telangiectasia hemorrágica hereditaria².

La telangiectasia hemorrágica hereditaria $(\mathrm{THH})$ 0 enfermedad de Rendu-Osler-Weber fue descrita por primera vez en el año 1876, por John Wickham Legg y, posteriormente, por Henri Jules Rendu, en 1896. En el año 1901, William Osler describió 3 pacientes que padecían una forma familiar rara de epistaxis recurrente, asociada con dilatación de los vasos sanguíneos de tamaño muy pequeño que daba lugar a manchas de color púrpura con aspecto de araña en piel y mucosas ${ }^{3}$. Weber en Londres (1906) describió casos similares de pacientes. Finalmente, en 1909, Hanes acuñó el término de THH en relación a estas tres características típicas del síndrome 4 .

Se trata de una alteración vascular displásica multisistémica caracterizada por la presencia de telangiectasias y malformaciones arteriovenosas (MAV) o conexiones directas que predisponen a la comunicación arteriovenosa y a la hemorragia ${ }^{5}$. Es una enfermedad genética de herencia autosómica dominante cuya prevalencia media es de 1 caso por cada 3.000-8.000 individuos, afectando a hombres y mujeres por edad sin predilección por sexo $0^{6}$.

Fue descrita inicialmente como una enfermedad caracterizada por sangrados nasales y gastrointestinales severos y recurrentes, asociados con anemia, vasos sanguíneos visibles y dilatados (telangiectasia), pudiendo presentar malformaciones arteriovenosas a nivel pulmonar, hepáticas, gastrointestinales, cerebrales, pancreáticas, y espinales $^{7,8}$.
La epistaxis es la manifestación clínica más común, encontrándose hasta en el $95 \%$ de los pacientes, se caracteriza por ser impredescible, variable en frecuencia, duración e intensidad ${ }^{9}$.

El espectro de la enfermedad es aún mayor, evidenciando asociación con otras patologías, tales como hipertensión arterial, poliposis juvenil (intestinal), hipertensión pulmonar, la cual a su vez es secundaria a una insuficiencia cardiaca derecha debido a malformaciones arterio-venosas hepáticas. Además, se ha visto un estado protrombótico en estos pacientes asociándose una elevación del factor VIII de la coagulación y potencial inmunodeficiencia ${ }^{10}$.

Como se mencionó anteriormente, esta es una enfermedad de herencia autosómica, donde se han identificado tres principales genes mutados asociados: Endoglina (ENG) correspondiente a una mutación en el cromosoma 9 que se atribuye a $25 \%-57 \%$ de los casos de HHT; ACRVL1/ALK1 también en el cromosoma 9, presente en 39\%$59 \%$ y SMAD4 en el cromosoma 12, asociada a $1 \%-2 \%$ de los casos ${ }^{10,11}$.

Estas mutaciones genéticas se traducen en la incapacidad de un vaso sanguíneo para madurar apropiadamente. Cada una se ha asociado a cierto genotipo de la enfermedad y mayor prevalencia de ciertas manifestaciones clínicas en cada una ${ }^{10}$ :

- Mutaciones en ENG corresponde a HHT 1, asociada con mayor frecuencia a MAV pulmonares ( $85 \%$ vs $35 \%$ HHT 2) y cerebrales. Las MAVs pulmonares generalmente son detectadas durante la pubertad, la mayoría no presenta síntomas respiratorios y menos de un tercio presenta signos sugerentes de shunt izquierda-derecha como cianosis 0 policitemia ${ }^{12,13}$.

- ACRVL1/ALK1 con HHT 2, destacando la mayor prevalencia de telangectasias cutáneas y MAV hepáticas y pancreáticas. Las telangectasias se generan por una desorganización de la adventicia, lo que lleva a dilatación de vasos, paredes adelgazadas y segmentos cortos. Estas no aparecen desde el nacimiento, sino que se desarrollan con la edad o durante el embarazo y pueden progresar e incluso regresar durante el transcurso de la enfermedad ${ }^{10}$.

- En el caso de SMAD4 destaca su asociación con poliposis juvenil ${ }^{10}$. 
El diagnóstico de THH es clínico. De acuerdo a Ios "Criterios de Curaçao" mencionados en la Tabla 1 , se tiene un diagnóstico seguro con $\geq 3$ criterios, diagnóstico sospechoso o posible si hay presente 2 criterios o diagnóstico indeterminado si tan solo encontramos 1 criterio $0^{4,14}$.

\section{CASO CLINICO}

Se presenta el caso de un paciente de 46 años, sexo masculino. Con antecedentes de hipertensión arterial, sin historia de traumatismos en zona facial. Refiere consumo del alcohol ocasional, tabaquismo de 2 cigarros por día hace 20 años, niega consumo de sustancias ilícitas. Refiere que madre y hermanos han presentado cuadros severos de epistaxis a repetición que han requerido transfusión.

Tabla 1. Criterios diagnósticos de THH

\begin{tabular}{|lr|}
\hline Criterio de Curaçao & \% de presentación \\
\hline 1. Epistaxis & $90-95$ \\
2. Telangiectasia & 80 \\
3. Lesiones Viscerales & \\
a) Gastrointestinal & $15-30$ \\
b) MAV pulmonar & 50 \\
c) MAV cerebrales & $10-20$ \\
d) MAV hepáticas & $30-70$ \\
e) MAV Espinales & $<1$ \\
4. Historia familiar & \\
\hline
\end{tabular}

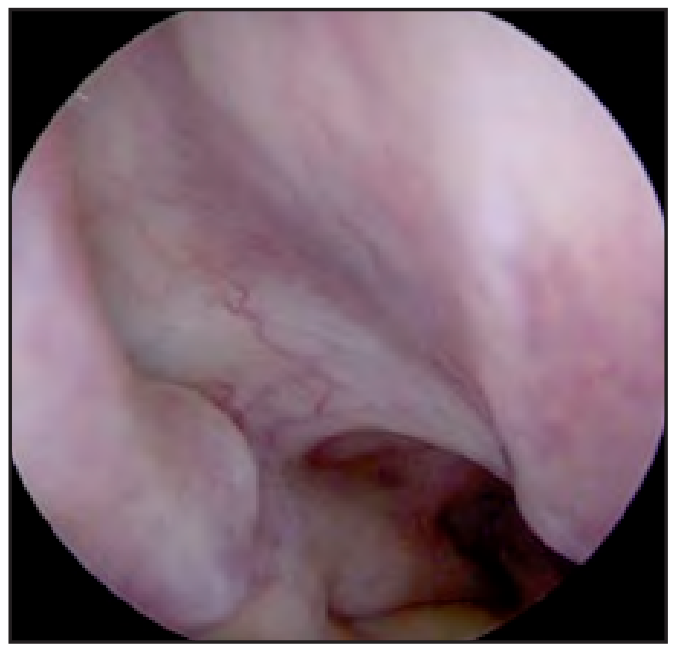

Figura 1. Mucosa telangiectásica a nivel de coana derecha.
Consulta de forma reiterada durante el año 2017 en Servicio de Urgencia del Hospital Carlos van Buren por epistaxis, cediendo con compresión digital, taponamiento anterior y cauterización química con nitrato de plata. Sin embargo en abril/2017 presenta episodio de epistaxis de gran cuantía que requiere transfusión de 2 unidades de glóbulos rojos, (debido a inestabilidad hemodinámica y hemoglobina sérica de $6,8 \mathrm{mg} /$ $\mathrm{dL}$ ). Se realiza taponamiento nasal anterior con gasa parafinada (Jelonet ${ }^{\circledR}$ ) y es hospitalizado en el servicio de otorrinolaringología para su estudio y manejo.

A las $72 \mathrm{hrs}$. se retira taponamiento nasal anterior presentando inmediatamente episodio de epistaxis severa, decidiéndose nuevo taponamiento nasal e ingreso de paciente a pabellón de urgencia para ligar arteria esfenopalatina.

Se ingresa por fosa nasal derecha bajo visión endoscópica con óptica de $0^{\circ}$, identificando mucosa nasal con abundantes telangiectasias dispersas por septum nasal, coanas y cornetes nasales (Figura 1).

Se identifica raíz del cornete medio, se realiza colgajo subperióstico y se identifica arteria esfenopalatina, la cual es ligada utilizando dos clips. Durante procedimiento, debido a las abundantes telangiectasias, se sospecha de la enfermedad de Rendu Osler Weber, tomando biopsia de la mucosa nasal (Figura 2).

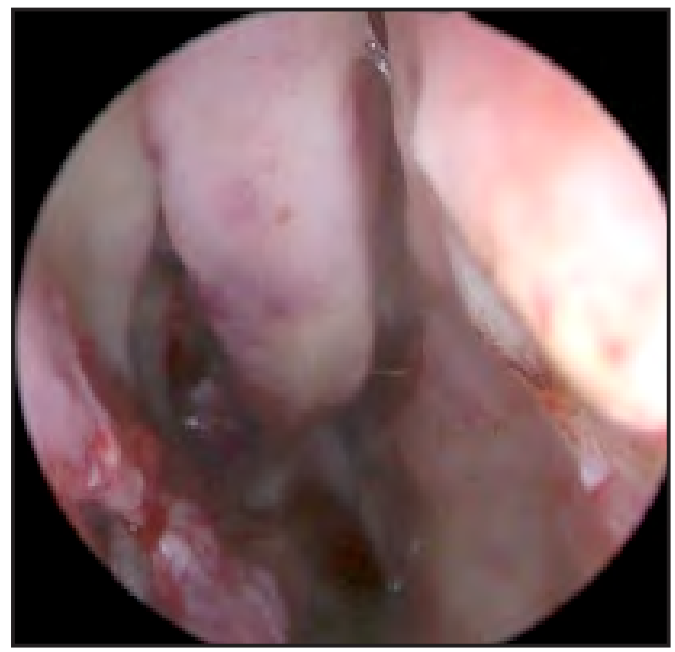

Figura 2. Toma de biopsia mucosa telangiectásica cornete inferior derecho. 
Se coloca taponamiento con gasa parafinada, sin presentar incidentes durante acto quirúrgico y se indica, además, ácido tranexámico (1 gr cada 8 hrs E.V por 2 días). Al cuarto día posquirúrgico se retiran tapones sin presentar nuevos episodios de epistaxis hasta la actualidad.

La biopsia es informada como rinosinusitis crónica atrófica leve con presencia de telangiectasias sugerentes de una enfermedad de Rendu Osler Weber (Figura 3).

Por antecedentes familiares, clínica del paciente y hallazgos de telangiectasia en mucosa nasal se plantea el diagnóstico de enfermedad de Rendu Osler Weber derivándose a neurología y medicina interna para descartar compromiso multisistémico de la enfermedad.

\section{DISCUSION}

En este artículo se presenta el caso de un paciente de 46 años que consulta por epistaxis y dado a sus antecedentes previos y a la evolución del cuadro, se sospecha THH.

En relación a los elementos clínicos de consenso internacional -Criterios de Curaça0 ${ }^{4,14}$-, para tener un diagnóstico seguro de THH se requiere la presencia de tres o más de los criterios mencionados con anterioridad.

En este caso encontramos epistaxis espontánea recurrente que desencadenó un estado anémico, telangiectasias nasales vistas al examen físico y con biopsia compatible e historia familiar de madre y hermanos con episodios similares, sin comprobación a la fecha de MAV viscerales. Ante estos antecedentes, y de manera retrospectiva, se estableció el diagnóstico seguro de THH.

Con respecto a las manifestaciones clínicas, la literatura describe que hasta $90 \%-95 \%$ de los casos de THH presentan episodios de sangrado de origen nasal principalmente sobre los 45-50 años de edad, cuya principal complicación es la presencia de anemia, tal como se evidenció en este paciente, requiriendo incluso transfusión de hemoderivados. Por otra parte, se describe que hasta $80 \%$ de los pacientes presentan telangiectasias mucocutáneas, siendo la mucosa nasal el lugar de presentación en este caso ${ }^{10,13}$.

A modo general, el manejo de un paciente con epistaxis considera de manera inicial, la estabilización hemodinámica y la compresión bidigital, seguido de una serie de medidas de acuerdo al grado de respuesta en cada situación clínica, tales como la cauterización, taponamiento nasal anterior, taponamiento nasal anterior bilateral, taponamiento nasal posterior, ligadura endoscópica y embolización arterial ${ }^{15}$.

El hecho de haber realizado el diagnóstico de manera retrospectiva, implicó que este paciente se manejara en una primera instancia tal como se habría hecho en cualquier caso de epistaxis en el servicio de urgencias según las recomendaciones internacionales, pero que no serían la primera línea de tratamiento en pacientes con diagnóstico
Figura 3. Biopsia que evidencia vasos telangiectásicos con elementos figurados en su interior y pared celular endotelial dilatada.

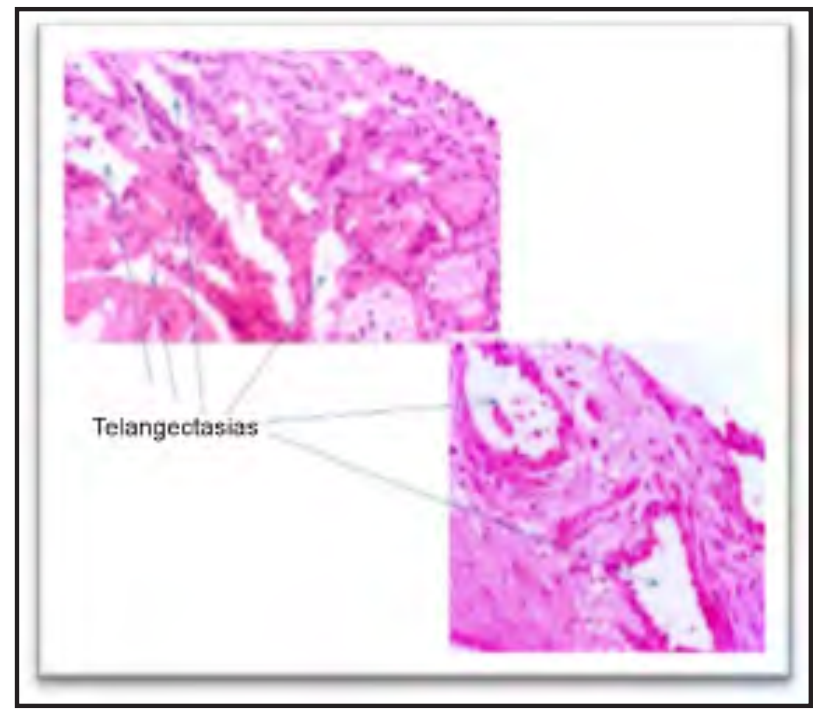


seguro de THH, según lo que se describe a continuación.

Dentro de las medidas recomendadas para el manejo específico de los episodios de epistaxis en pacientes con THH publicadas recientemente por la Sociedad Francesa de Otorrinolaringología y Cirugía de Cabeza y Cuello ${ }^{6}$, se encuentra la compresión bidigital y en caso de no obtener una respuesta, el taponamiento nasal con material reabsorbible asociado a antibioterapia profiláctica. En casos resistentes a estas medidas locales que presenten caída del hematocrito, se podría realizar embolización arterial (A. maxilar y A. facial) y uso de ácido tranexámico o bevacizumab9,16. La utilización de taponamiento con material no reabsorbible y la cauterización ya sea química o eléctrica no se recomiendan como medidas de enfrentamiento de primera línea. Ante la presencia de telangiectasias nasales demostradas, se puede utilizar inyección de sustancias esclerosantes, gel hemostático y ablación con láser y/o radiofrecuencia. En cuanto a las intervenciones quirúrgicas la dermoplastía septal no se recomienda, y como último recurso podría realizarse un cierre narinal utilizando la técnica de Young9,17.

Luego del manejo agudo de la hemorragia en pacientes con diagnóstico de THH, se debería realizar un estudio multidisciplinario, comprendido por evaluación de la anemia, búsqueda de MAV pulmonares, y en algunos casos cerebrales, hepáticas y espinales ${ }^{10,18}$, etapa en la que se encuentra actualmente el paciente del caso descrito.

\section{BIBLIOGRAFÍA}

1. Alfredo Naser G., Cristián Aedo B. Epistaxis: diagnóstico y alternativas terapéuticas actuales Rev. Hosp. Clín. Univ. Chile 2007; 18; 227-38.

2. René Esteban Moreno Rajadel, Armando J. Figueroa Hernández y Alejandro díaz González. Epistaxis: Consideraciones sobre aspectos clínicos y terapéuticos en la atención primaria de salud. Rev Cubana Med Gen Integr v.23 n.4 Ciudad de La Habana oct.-dic. 2007.

3. Gloria Astencio Rodríguez; Onix César Garib Alpízar; José napoleón Ventura Blanco; Boris l. Torres Cueva; (†) Nancy de León Rubio; Jorge Luis
En cuanto al estudio genético, no está recomendado, basta la aproximación a través de los criterios clínicos mencionados con cifras aceptables de VPP de $100 \%$ y VPN de $97 \%{ }^{14}$.

\section{CONCLUSIÓN}

La telangiectasia hemorrágica familiar se debe sospechar y tener en consideración en pacientes mayores de 40 años con sangrado nasal recurrente y/o asociado a anemia, siempre teniendo en cuenta que abarca múltiples sistemas de órganos y dentro de estos mismos con numerosas formas de enfermedad.

A pesar que el diagnóstico de la enfermedad requiere en su mayoría elementos clínicos sin el apoyo fundamental de exámenes complejos y de alto costo permanece en su mayoría subdiagnosticada.

Implica una amplia de disciplinas científicas (laboratorio, imagenología, genética entre otras) y clínicas (otorrinolaringología, neurología, medicina interna con sus diferentes subespecialidades, entre otras) siendo fundamental para poder manejar esta patología el apoyo multidisciplinario.

Corresponde a un trastorno muy desafiante para comprender genéticamente, existiendo aún más genes involucrados que están en estudios y que podrían permitir a futuro una terapia más individualizada según los mecanismos génicos involucrados.

Hernández. Rendu-Osler-Weber síndrome. Rev cubana med v.48 n.3 Ciudad de la Habana jul.sep. 2009.

4. Andrés Alvo V, Eugenio Alzérreca A. Telangiectasia hemorrágica hereditaria: Aspectos otorrinolaringologías Rev. Otorrinolaringol. Cir. Cabeza Cuello vol.72 no.3 Santiago dic. 2012; 297-305.

5. Di Cosola M, Cazzolla AP, Scivetti M, Testa NF, lo Muzio L, Favia G Carrillo de Albornoz A, Bascones A. Síndrome de Rendu-Osler-Weber - Telangiectasia Hemorrágica Hereditaria (HHT): Descripción de dos casos y revisión de la literatura. Av Odontoestomatol vol. 21 no.6 Madrid nov./dic. 2005. 
6. O. Ramírez Balzaa, L. García-Guereta, M.D. Rubio Vidal, M.J. del Cerro Marín, M. Parrón Pajares Síndrome de Rendu-Osler-Weber: causa infrecuente de hipoxemia en la infancia. An Pediatr 2010; 73: 272-6 - Vol. 73 Núm.5 DOI: 10.1016/j.anpedi.2010.06.007.

7. Geisthoff UW, Maune S, Schneider G. Hereditary hemorrhagic telangiectasia (Rendu-OslerWeber disease) as an example of a rare disease relevant for oto-rhino-laryngology. Laryngorhinootologie 2011; 90(4): 230-42.

8. Jason E. Cohn, D01 and Joshua Scharf, MD2 Telangiectasias in Osler-Weber-Rendu Syndrome Otolaryngology- Head and Neck Surgery 1-2 American Academy of Otolaryngology-Head and Neck Surgery Foundation 2017.

9. L. Robarda, J. Michelb, V. Prulière Escabassec, E. Bequignonc, B. Vérillaudd, O. Malarde, L. Crampettef. Recommandation de la SFORL (version courte). Prise en charge spécifique des épistaxis dans le cadre d'une maladie de Rendu-Osler Annales françaises d'Oto-rhinolaryngologie et de Pathologie Cervico-faciale, Volume 134, Issue 1, February 2017, Pages 34-8.

10. ClaIRE L. Shovlin Hereditary haemorrhagic telangiectasia: Pathophysiology, diagnosis and treatment NHLI Cardiovascular Sciences, Imperial College London, UK and HHTIC London, Hammersmith Hospital, Imperial College Healthcare NHS Trust, London, UK 2010.

11. McDonald J, PyeRItz RE. Hereditary Hemorrhagic Telangiectasia. 2000 Jun 26 [Updated 2017 Feb 2]. In: Pagon RA, Adam MP, Ardinger $\mathrm{HH}$, et al., editors. GeneReviews ${ }^{\circledR}$ [Internet]. Seattle (WA): University of Washington, Seattle; 1993-2017.

12. Shovlin CL, JaCkson JE, Bamford KB, et al. Primary determinants of ischaemic stroke/brain abscess risks are independent of severity of pulmonary arteriovenous malformations in hereditary haemorrhagic telangiectasia. Thorax 2008; 63: 259.M E.

13. Begbie, GMF Wallace, C L Shovlin. Hereditary haemorrhagic telangiectasia (Osler-WeberRendu syndrome): a view from the $21^{\text {st }}$ century. Postgrad Med J 2003; 79: 18-24.

14. Van Gent MWF, Velthuis S, Post MC, Snijder RJ, Westermann CJJ, Letteboer TGW, Mager JJ. 2013. Hereditary hemorrhagic telangiectasia: How accurate are the clinical criteria? Am J Med Genet Part A 161A: 461-6.

15. Zachary A. Kasperek, Gary F. Pollock. Epistaxis: An Overview. Emerg Med Clin N Am 31 (2013) 443-54.

16. Cameron Grigg, MD, Daniel Anderson, MD, James EARnshaw, MD. Diagnosis and Treatment of Hereditary Hemorrhagic Telangiectasia Ochsner Journal 17: 157-161, 2017, Australia.

17. Nathan B. Sautter, Timothy L. Smith. Treatment of Hereditary Hemorrhagic Telangiectasia-Related Epistaxis. Otolaryngol Clin N Am 49 (2016) 63954.

18. ME Faughnan, Va Palda, G Garcia-Tsao, UW Geisthoff, J McDonald, DD Proctor, J Spears, DH Brown, E Buscarini, MS Chesnutt, V Cottin, A Ganguly, JR Gossage, AE Guttmacher, RH Hyland, SJ Kennedy, J Korzenik, JJ Mager, AP Ozanne, JF Piccirillo, D Picus, H Plauchu, Mem Porteous, RE Pyeritz, DA Ross, C Sabba, K Swanson, P Terry, mC Wallace, CJJ Westermann, RI White, LH Young, R Zarrabeitia. International guidelines for the diagnosis and management of hereditary haemorrhagic telangiectasia. J Med Genet2011; 48: $73 \mathrm{e} 87$. 